\title{
CFTR modulators: transformative therapies for cystic fibrosis
}

\author{
Mary Dwight and Bruce Marshall, MD
}

Advances in treatment and care have transformed cystic fibrosis (CF). Once considered a pediatric disease, today more than half of all people with $\mathrm{CF}$ are over the age of 18 years. With gains in life expectancy, people with $\mathrm{CF}$ are realizing milestones such as graduating from high school or college, starting families, and pursuing careers. At the same time,

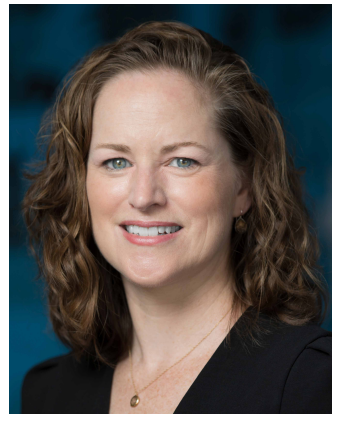

Mary Dwight

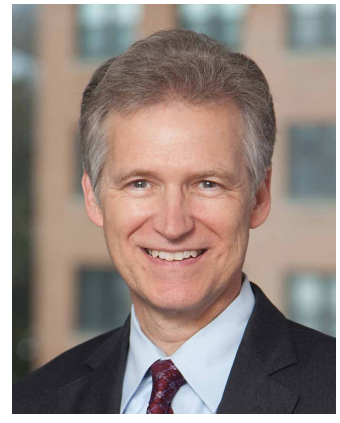

Bruce Marshall, MD the progressive nature of the disease means people with $\mathrm{CF}$ are also experiencing worsening manifestations as they age, requiring symptomatic maintenance to delay life-threatening infections and irreversible lung damage every day.,

The most transformative advance in $\mathrm{CF}$ treatment has been the availability of highly effective modulators that target the underlying defect in the CFTR protein caused by mutations of the CFTR gene. Because modulator therapy targets the underlying cause of $\mathrm{CF}$, benefits are seen in multiple organ systems. Modulator therapy is associated with improvements in key measures of disease severity, including lung function, body mass index, the rate of pulmonary exacerbations, and quality of life. ${ }^{3}$ On a daily basis, these medicines enable patients to experience life events that most people take for granted, such as carrying their child to bed or running a mile without stopping to cough.
The first highly effective modulator therapy, Kalydeco (ivacaftor), was approved by the U.S. Food and Drug Administration (FDA) in 2012 for about $4 \%$ of people with CF with specific gating mutations in the CFTR gene. ${ }^{4}$ Three additional modulators have been approved since, including Trikafta (elexacaftor/tezacaftor/ ivacaftor) in October 2019, greatly expanding the eligible population for this class of drugs. With clinical studies underway in children aged under 12 years, Trikafta has the potential to benefit approximately $90 \%$ of people with $\mathrm{CF}$ in the United States, meaning for the first time, most people living with $\mathrm{CF}$ will be eligible for a life-changing treatment. ${ }^{5}$

The high cost associated with treating CF has spurred important conversations about clinical effectiveness and cost-effectiveness. Recognizing the significant clinical

\section{Author affiliations}

Mary Dwight and Bruce Marshall, MD, Cystic Fibrosis Foundation, Bethesda, MD.

AUTHOR CORRESPONDENCE:

Mary Dwight, 240.482.2871;

mdwight@cff.org

J Manag Care Spec Pharm. 2021;27(2):281-84

Copyright $\odot 2021$, Academy of Managed Care Pharmacy. All rights reserved.

effect of modulators, the Institute for Clinical and Economic Review (ICER) awarded Trikafta an "A," its highest grade for clinical effectiveness and highlighted the "high certainty that the treatment delivers substantial health benefits." ${ }^{\prime \prime}$ ICER's report ("Modulator Treatments for Cystic Fibrosis: Effectiveness and Value") also underscored the importance of these therapies and their role in the broader CF care regimen, noting that "access to Trikafta is not and should not be viewed as negotiable." ${ }^{6}$ We agree: all eligible patients should have these life-saving treatments.

Given the progressive nature of $\mathrm{CF}$ and clear evidence regarding which mutations respond to modulator therapy, insurance benefit design should support access for individuals of 


\section{FIGURE 1 Intravenous-Treated Pulmonary Exacerbations Following Trikafta According to the CFF Patient Registry}

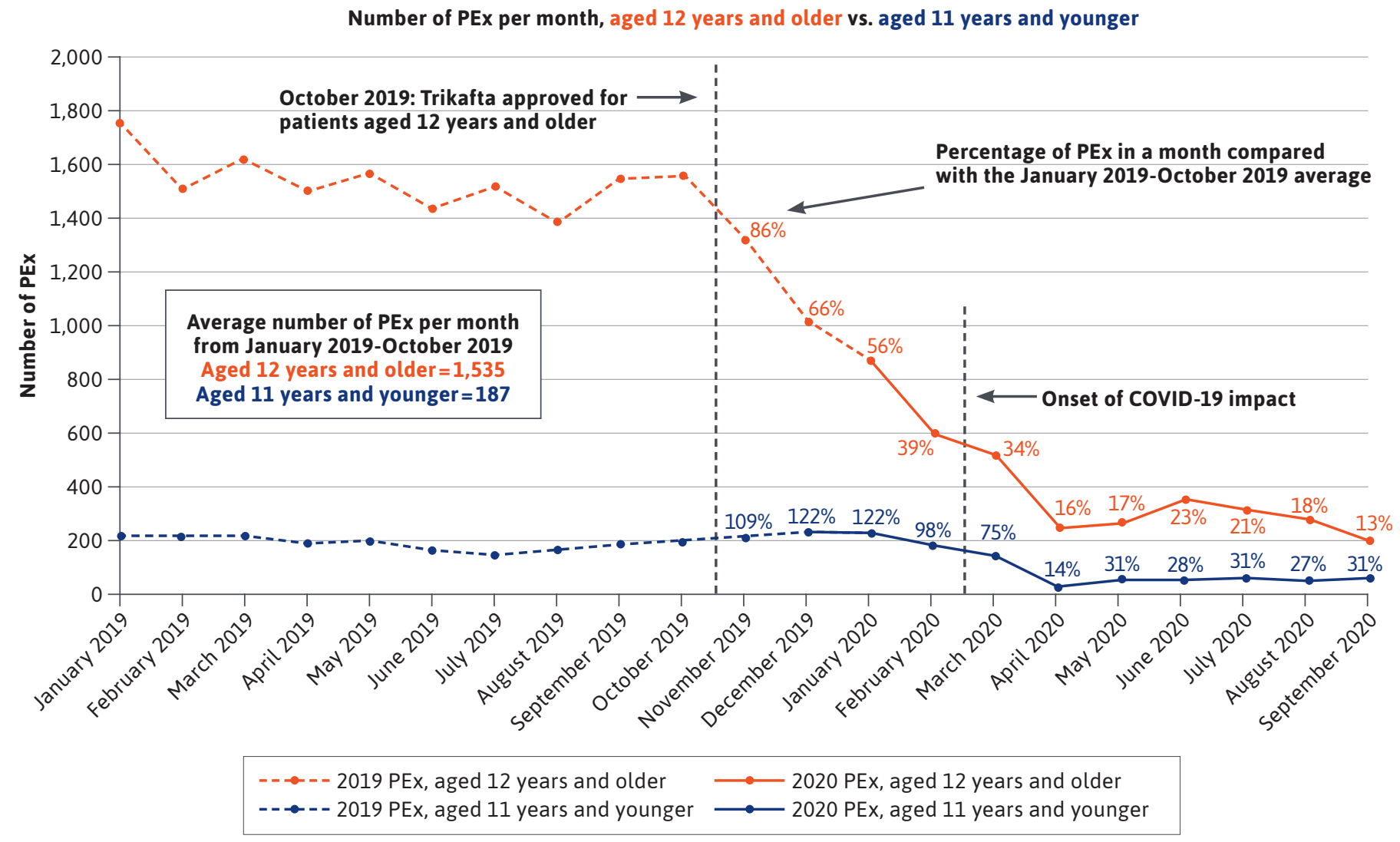

Note: Data entry into the CFF Patient Registry is not complete for 2020, and this data may change upon locking of the reporting year. This chart reflects all data entered into the registry by September 30, 2020.

$\mathrm{CFF}=$ Cystic Fibrosis Foundation; $\mathrm{PEX}=$ acute pulmonary exacerbation.

eligible age with eligible mutations without undue administrative or financial burden. Coverage criteria must be clear and clinically appropriate on initial authorizations and reauthorizations. One example of clinically inappropriate criteria is requirements for patients to undergo continual genetic testing to prove they still have $\mathrm{CF}$ or a relevant mutation-a disease that presently has no cure. Similarly, requiring patients to "fail" on a symptom-directed therapy to obtain a therapy that actually corrects the underlying cause of CF unnecessarily delays access to these first-line treatments. These examples are egregious and highlight the burden shouldered by people with $\mathrm{CF}$ and their care providers to obtain these medically necessary therapies. It is also critical that payers prioritize swift authorization of these drugs for eligible patients. Delays in access can lead to irreversible disease progression, thereby altering an individual's course of disease and causing worsening symptoms.

Furthermore, patients eligible for modulators must not be subject to out-of-pocket costs that could preclude access. Of particular concern is the use of accumulator adjustment programs that require patients with costly care regimens to shoulder even more of the financial burden to access necessary medications. These programs disproportionately affect patients who take multiple drugs, such as people with CF. A small, incremental increase in cost may seem trivial from the payer perspective, but the totality of costs for the CF care regimen can snowball into an unsustainable burden for the person with CF. Strategies to contain costs that impede patient access to a life-saving treatment are unacceptable. 
In the year that Trikafta has been available, it has already proved to be a life-changing drug. According to the CF Foundation Patient Registry, which collects health information from consented patients who receive care at a CF Foundation-accredited care center, pulmonary exacerbations (a sudden worsening of pulmonary symptoms characterized by intravenous antibiotic treatment) have dramatically decreased following the approval of Trikafta (Figure 1). ${ }^{2}$ Early data indicate that the long-term potential of modulators may transform the disease's trajectory. Thus, it is important to note that although ICER evaluated currently available research, long-term data are not yet available. Key unknowns include the effects of long-term modulator use and early initiation of therapy. People with CF who start modulator treatment at a young age may be able to restore CFTR protein function to near normal levels, thus, preventing organ damage and halting progression of the disease. ${ }^{7}$

In the future, Trikafta will likely become available to younger patients, allowing for early initiation of this therapy and profound long-term benefits due to the prevention of damage to the lungs, pancreas, liver, sinuses, and other organs that lead to devastating clinical manifestations of the disease. ${ }^{8,9}$ For these people, we anticipate a life span that approximates that of the general population and that eventually most, if not all, of the cost associated with current standard-ofcare treatments might be eliminated. However, it is important to note that patients whose disease has progressed significantly before accessing a modulator will still require access to symptom-directed treatments.
Studies are underway to evaluate the effect of withdrawing symptomdirected therapies in patients taking Trikafta. ${ }^{10}$

Despite the progress of studies to address the unknowns of long-term and real-world effects of modulators on health status, quality of life, resource utilization, and other factors, we recognize that payers need to make coverage decisions todaywithout these data. ${ }^{11,12}$ Aligning access with the FDA label becomes even more important given the benefits being realized today and the long-term potential of these therapies. ICER's report must only be used as part of a holistic evaluation of the value a treatment provides and the value of that treatment within the larger treatment regimen. We urge managed care decision makers to heed ICER's advice that "public and private payers should continue to affirm their commitment to provide access to the CFTR modulators and should remove superfluous requirements for coverage approval and continuation." 5

Short of a cure for CF, modulators are the greatest opportunity to dramatically alter the course of this chronic, life-shortening disease. As the first and best treatment option to address this complex, multisystem disease, people with CF must have access to the most effective modulator available for their genetic mutation. As cost-effectiveness discussions continue and coverage decisions are made, it is critical to reflect on the dramatic effect these therapies have already had for so many and their anticipated long-term value, and, above all, to keep the patient at the center of every decision.

\section{DISCLOSURES}

No funding contributed to the writing of this commentary. Both authors are employed by the Cystic Fibrosis Foundation. The Cystic Fibrosis Foundation has entered into therapeutic development award agreements and licensing agreements to assist with the development of CFTR modulators that may result in intellectual property rights, royalties, and other forms of consideration provided to CFF. Some of these agreements are subject to confidentiality restrictions and, thus, CFF cannot comment on them.

\section{REFERENCES}

1. Cystic Fibrosis. Centers for Disease Control and Prevention. Cystic fibrosis. May 18, 2020. Accessed January 9, 2021. https://www.cdc.gov/genomics/disease/ cystic_fibrosis.htm

2. Cystic Fibrosis Foundation. 2019 Patient Registry Annual Data Report. 2020.

Accessed January 9, 2021. https://www. cff.org/Research/Researcher-Resources/ Patient-Registry/2019-Patient-RegistryAnnual-Data-Report.pdf

3. Middleton PG, Mall MA, Dřevínek P, et al. Elexacaftor-tezacaftor-ivacaftor for cystic fibrosis with a single Phe508del allele. N Engl J Med. 2019;381(19):1809-19. doi: 10.1056/NEJMoa1908639

4. Kalydeco (ivacaftor) tablets, for oral use. Vertex Pharmaceuticals. Revised May 2017. Accessed January 9, 2021. https:// www.accessdata.fda.gov/drugsatfda docs/label/2017/203188s019lbl.pdf

5. U.S. Food and Drug Administration. FDA approves new breakthrough therapy for cystic fibrosis. October 21, 2019. Accessed January 9, 2021. https://www.fda.gov/ news-events/press-announcements/ fda-approves-new-breakthrough-therapycystic-fibrosis

6. Institute for Clinical and Economic Review. Modulator treatments for cystic fibrosis: effectiveness and value. Final evidence report and meeting summary. September 23, 2020. Accessed January 9, 2021. http://icerorg.wpengine.com/ wp-content/uploads/2020/08/ICER_ CF_Final_Report_092320.pdf 
7. Rosenfeld M, Wainwright CE, Higgins M, et al. Ivacaftor treatment of cystic fibrosis in children aged 12 to $<24$ months and with a CFTR gating mutation (ARRIVAL): a phase 3 single-arm study [published correction appears in Lancet Respir Med. 2018;6(7):e35; published correction appears in Lancet Respir Med. 2019;7(4):e15]. Lancet Respir Med. 2018;6(7):545-53. doi: 10.1016/ S2213-2600(18)30202-9

8. Sawicki GS, McKone EF, Pasta DJ, Millar SJ, et al. Sustained benefit from ivacaftor demonstrated by combining clinical trial and cystic fibrosis patient registry data. Am J Respir Crit Care Med. 2015;192:836-42.
9. Konstan MW, McKone EF, Moss RB, et al. Assessment of safety and efficacy of long-term treatment with combination lumacaftor and ivacaftor therapy in patients with cystic fibrosis homozygous for the F508del-CFTR mutation (PROGRESS): a phase 3, extension study. Lancet Respir Med. 2017;5(2):107-18. doi: 10.1016/S2213-2600(16)30427-1

10. Cystic Fibrosis Foundation. Clinical trial finder. SIMPLIFY: Study to evaluate stopping inhaled hypertonic saline or dornase alfa in people with CF who are taking the triple-combination modulator, elexacaftor/tezacaftor/ ivacaftor (SIMPLIFY-IP-19). Accessed January 9, 2021. https://www.cff. org/Trials/Finder/details/580/ SIMPLIFY-Study-to-evaluate-stoppinginhaled-hypertonic-saline-or-dornasealfa-in-people-with-CF-who-are-takingthe-triple-combination-modulator-elexacaftor-tezacaftor-ivacaftor
11. Cystic Fibrosis Foundation. CFF trial finder. Study to evaluate the effects of the triple-combination modulator, elexacaftor/tezacaftor/ivacaftor (PROMISE) (PROMISE-OB-18). Accessed January 9, 2021. https://www.cff. org/Trials/Finder/details/540/ Study-to-evaluate-the-effects-ofthe-triple-combination-modulatorelexacaftor-tezacaftor-ivacaftor-PROMISE

12. Cystic Fibrosis Foundation. CFF trial finder. Study to evaluate the effects of CFTR modulators in infants and young children (BEGIN Part B; BEGIN-OB-19 Part B). Accessed January 9, 2021. https:// www.cff.org/Trials/Finder/details/627/ Study-to-evaluate-the-effects-of-CFTRmodulators-in-infants-and-youngchildren-BEGIN-Part-B 